\title{
NUMERICAL STUDY OF FLOW IN AXISYMMETRIC TURBULENT OPPOSED JETS
}

\author{
A. Abdel- Fattah \\ Department of Mechanical Power Engineering, Faculty of Engineering Minoufiya \\ University, Shebin El-Kom, Egypt. \\ ashourabdelfatah@yahoo.com
}

\begin{abstract}
In this study the numerical investigation for unsteady two dimensional of turbulent opposed jets is presented. The numerical study is based on the solution of the complete Navier - Stokes equations and turbulence models using a finite volume technique. TDMA solver is used to solve the algebraic equations. The pressure - velocity coupling is achieved using the SIMPLE method. Simulation is carried out for a range of Reynolds number $5000 \leq \operatorname{Re} \leq 11700$. The distance between the exit of two nozzles is $0.4 \leq \mathrm{H} / \mathrm{d} \leq 1.0$. The results show that the radial velocity increases in downstream direction until maximum and then decreases. This change depends on the distance between the nozzles exit and the flow time. The axial velocity decays in the down stream direction on the axial direction. The turbulence kinetic energy and the normal stress in the axial direction increase and they become a maximum at the stagnation point, which occurs in the separation plane. The pressure coefficient at the stagnation point decays in downstream direction. This decay decreases by increasing the distance between the nozzles. The comparison between the numerical results and previous experimental measurements gives better agreement for $\bar{v}^{2}-\mathrm{f}$ turbulence model than RNG model and $\mathrm{k}-\varepsilon$ standard model.
\end{abstract}

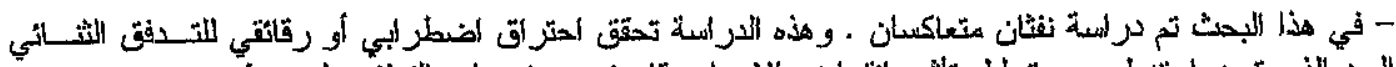

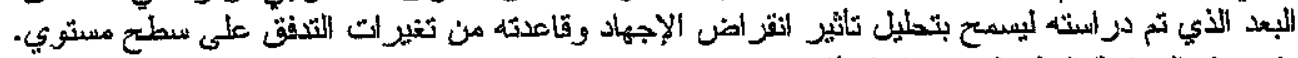

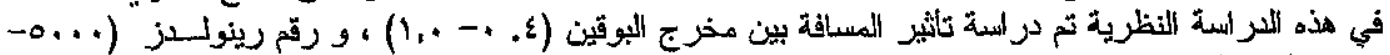

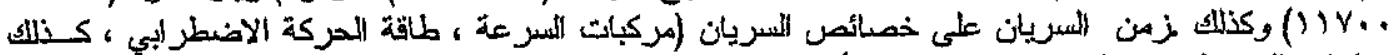

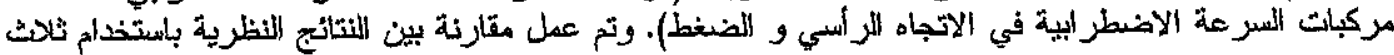

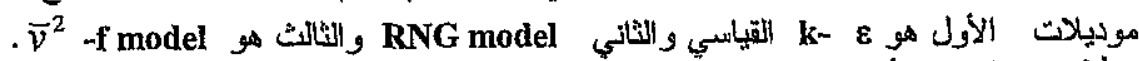
وأظهرت الفناتج الأتي:

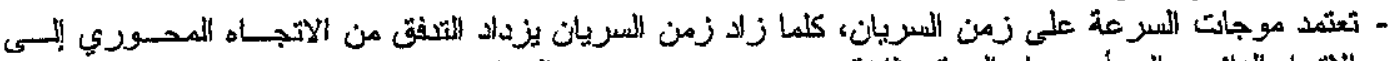

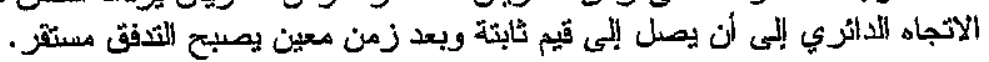

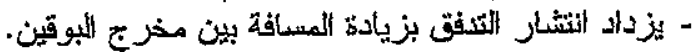

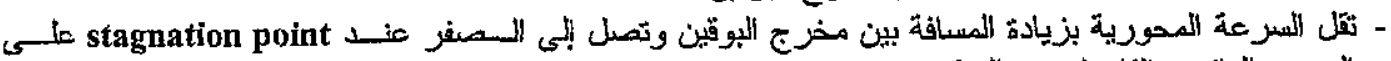

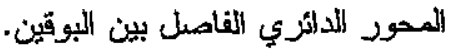

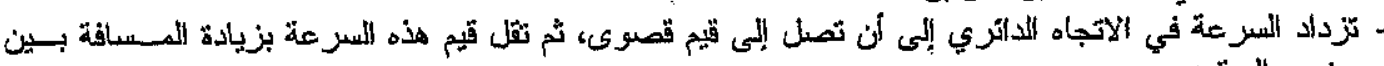
مخرج البوقين.

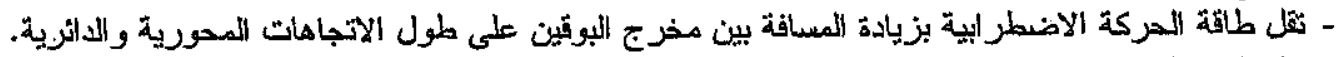

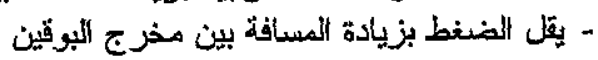

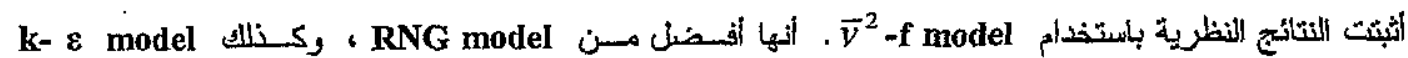
(1) standard

Keywords; opposed jets; $\bar{v}^{2}$-f turbulence model; RNG model; k- $\varepsilon$ standard model; turbulent flow

\section{INTRODUCTION}

Opposed jets with fuel and air can be used for the investigation of laminar or turbulent combustion. The flow produced is two-dimensional allows analysis of the effect of strain extinction and does not have the complications of flows that stagnate onto a flat plate. Some studies of the flow characteristics for opposed jets have been published. Velocity measurements in the isothermal flows created by opposed nozzle configuration indicated by Lindstedt et al.[1]. Their results showed the axial velocity decreases along the 


\section{MATHMATICAL MODELS}

The physical model used in this study is shown in Figure 1(a) The fluid is air and the flow field is two dimensional, incompressible and turbulent Based on the characteristics scales of $d$ and $V_{J}$ the dimensionless variables are defined as follows.

$$
\begin{gathered}
t=\frac{\bar{t} V_{J}}{d}, \quad r=\frac{\bar{r}}{d}, y=\frac{\bar{y}}{d}, \quad k=\frac{\bar{k}}{V_{J}^{2}}, \quad u=\frac{\bar{u}}{V_{J}}, \\
v=\frac{v^{\prime}}{V_{J}}, \quad p=\frac{\bar{p}}{0.5 \rho V_{J}^{2}}, \quad f=\frac{\bar{f} d}{V_{J}}, \quad \varepsilon=\frac{\bar{\varepsilon} d}{V_{J}^{3}}, \\
\tau=\frac{\bar{\tau} V_{J}}{d}, \quad v^{\prime}=\frac{\bar{v}}{V_{J} d}
\end{gathered}
$$

Where the over bar represents the dimensional quantities. According to the above assumptions and dimensionless variables, the dimensionless governing equations are expressed as the following equations.

Continuity equation

$\frac{1}{r}\left(\frac{\partial r u}{\partial r}+\frac{\partial r v}{\partial y}\right)=0$

\section{Radial momentum equations:}

$\frac{\partial u}{\partial t}+u \frac{\partial u}{\partial r}+v \frac{\partial u}{\partial y}=-\frac{\partial p}{\partial r}+\frac{1}{r} \frac{\partial}{\partial r}\left[r\left(\frac{1}{\operatorname{Re}}+\frac{1}{\operatorname{Re}_{t}}\right) \frac{\partial u}{\partial r}\right]$

$+\frac{\partial}{\partial y}\left[\left(\frac{1}{\operatorname{Re}}+\frac{1}{\operatorname{Re}_{t}}\right) \frac{\partial u}{\partial y}\right]+\frac{1}{r} \frac{\partial}{\partial r}\left(\frac{r}{\operatorname{Re}_{t}} \frac{\partial u}{\partial \dot{r}}\right)+\frac{\partial}{\partial y}\left(\frac{1}{\operatorname{Re}_{t}} \frac{\partial v}{\partial x}\right)$

$-\frac{2 u}{\operatorname{Re}_{\mathrm{t}} r^{2}}$

Axial momentum equations:

$$
\begin{aligned}
& \frac{\partial v}{\partial t}+u \frac{\partial v}{\partial r}+v \frac{\partial v}{\partial y}=-\frac{\partial p}{\partial y}+\frac{1}{r} \frac{\partial}{\partial r} \\
& {\left[r\left(\frac{1}{\operatorname{Re}}+\frac{1}{\operatorname{Re}_{t}}\right) \frac{\partial v}{\partial r}\right]+\frac{\partial}{\partial y}\left[\left(\frac{1}{\operatorname{Re}_{\mathrm{e}}}+\frac{1}{\operatorname{Re}_{t}}\right) \frac{\partial v}{\partial y}\right]} \\
& +\frac{1}{r} \frac{\partial}{\partial r}\left(\frac{r}{\operatorname{Re}_{t}} \frac{\partial u}{\partial y}\right)+\frac{\partial}{\partial y}\left(\frac{1}{\operatorname{Re}_{t}} \frac{\partial v}{\partial y}\right)
\end{aligned}
$$

The dimensionless equations for standard $\mathrm{k}-\varepsilon$ model are written as:

$$
\begin{aligned}
& \frac{\partial k}{\partial t}+u \frac{\partial k}{\partial r}+v \frac{\partial k}{\partial y}=\frac{1}{r} \frac{\partial}{\partial r}\left[r\left(\frac{1}{\operatorname{Re}}+\frac{1}{\sigma_{\varepsilon} \operatorname{Re}_{t}}\right) \frac{\partial k}{\partial r}\right] \\
& +\frac{\partial}{\partial y}\left[\left(\frac{1}{\operatorname{Re}}+\frac{1}{\sigma_{\varepsilon} \operatorname{Re}_{t}}\right) \frac{\partial k}{\partial y}\right]+G-\varepsilon
\end{aligned}
$$

$$
\begin{aligned}
& \frac{\partial \varepsilon}{\partial t}+u \frac{\partial \varepsilon}{\partial r}+v \frac{\partial \varepsilon}{\partial y}=\frac{1}{r} \frac{\partial}{\partial r}\left[r\left(\frac{1}{\operatorname{Re}}+\frac{1}{\sigma_{\varepsilon} \mathrm{Re}_{t}}\right) \frac{\partial \varepsilon}{\partial r}\right] \\
& +\frac{\partial}{\partial y}\left[\left(\frac{1}{\operatorname{Re}}+\frac{1}{\sigma_{\varepsilon} \operatorname{Re}_{t}}\right) \frac{\partial \varepsilon}{\partial y}\right]+c_{1} \frac{\varepsilon}{k} G-c_{2} \frac{\varepsilon^{2}}{k}
\end{aligned}
$$

Where $G$ is the rate of production of turbulent kinetic energy and is given by:

$$
G=\frac{2 S^{2}}{\mathrm{Re}_{t}}
$$

and

$$
S^{2}=\frac{1}{2}\left[\left(\frac{\partial u}{\partial y}+\frac{\partial v}{\partial r}\right)^{2}+2\left(\frac{\partial u}{\partial r}\right)^{2}+2\left(\frac{u}{r}\right)^{2}+2\left(\frac{\partial v}{\partial y}\right)^{2}\right]
$$

The values of the model constants as published in [7] are taken as:

$$
\begin{aligned}
& c_{1}=1.44, c_{2}=1.92, \quad c_{\mu}=0.09, \quad \sigma_{\mathrm{k}}=1 \text { and } \\
& \sigma_{\varepsilon}=1.3
\end{aligned}
$$

The renormalization group dissipation is given by:

$$
\begin{aligned}
& \frac{\partial \varepsilon}{\partial}+u \frac{\partial \varepsilon}{\partial t}+v \frac{\partial \varepsilon}{\partial y}=\frac{1}{r} \frac{\partial}{\partial}\left[r\left(\frac{1}{\operatorname{Re}}+\frac{1}{\sigma_{\varepsilon} \operatorname{Re}}\right) \frac{\partial \varepsilon}{\partial r}\right]+\frac{\partial}{\partial} \\
& {\left[\left(\frac{1}{\operatorname{Re}}+\frac{1}{\sigma_{\varepsilon} \operatorname{Re}}\right) \frac{\partial \varepsilon}{\partial y}\right]+\frac{\varepsilon}{k}\left(c_{1}-\frac{\eta\left(1-\frac{\eta}{\eta_{0}}\right)}{1+\beta \eta^{3}}\right) G-c_{2} \frac{\varepsilon^{2}}{k}} \\
& \text { where } \eta=\sqrt{c_{\mu}^{-1} \frac{G}{\rho \varepsilon}}
\end{aligned}
$$

The values of the model constants are taken as:

$$
\begin{aligned}
& c_{\mu}=0.085, c_{1}=1.44, c_{2}=1.68, \sigma_{k}=1.39, \\
& \sigma_{\varepsilon}=1.39, \eta_{0}=4.38 \text { and } \beta=0.012
\end{aligned}
$$

where $\mu_{t}$ is the eddy viscosity is given by the relation

$$
\mu_{t}=c_{\mu} \frac{\rho k^{2}}{\varepsilon}
$$

The dimensionless equation of dissipation equation for the $\vec{v}^{2}$-f model is written as;

$$
\begin{aligned}
& \frac{\partial \varepsilon}{\partial t}+u \frac{\partial \varepsilon}{\partial r}+v \frac{\partial \varepsilon}{\partial y}=\frac{1}{r} \frac{\partial}{\partial r}\left[r\left(\frac{1}{\operatorname{Re}}+\frac{1}{\sigma_{\varepsilon} \operatorname{Re}_{t}}\right) \frac{\partial \varepsilon}{\partial r}\right] \\
& +\frac{\partial}{\partial y}\left[\left(\frac{1}{\operatorname{Re}}+\frac{1}{\sigma_{\varepsilon} \operatorname{Re}_{t}}\right) \frac{\partial \varepsilon}{\partial y}\right]+\frac{c_{\varepsilon 1} G-c_{\varepsilon 2} \varepsilon}{\tau}
\end{aligned}
$$

The dimensionless equations for $\bar{v}^{2}$-f are given by: 
axial direction of the jets. Yang et al [2] studied the turbulent flow of impinging and countercurrent jets by Reynolds stress model (RSM). Their results are compared with those of the $\mathrm{k}-\varepsilon$ model and availate data to assess the flow characteristics and turbulence models. The variants of RSM tested were those of Gibson and Launder (GL), Graft and Launder ( GLCL) and speziale, Sakar and Gatski (SSG). The counter flow flames of air and methane, propone and ethylene with and without periodic force studied by Luff et al.[3].Their results showed that, the effect of strain rate on the nature of extinction and characteristic time scale of premixed turbulent flames, comprising air and each of methane, propane and ethylene. Solution of reduced forms of the Navier-Stokes equations with numerical and turbulence assumptions have been reported for impinging flow, usually those impinging on to surfaces, by several authors including Korvsoy and Whitelaw [4] ,Park and Sung [5] and Chattopadhyay [6]. Salmanzadeh et al. [7] used $\bar{v}^{2}$ f model to study the flow and thermal fields in an axisymmetric, fully developed, turbulent, impinging jet on a flat plate. The influence of disk rotation, the distance from nozzle to disk and Reynolds number on the flow characteristics of an impinging jet were studied numerically by Abdel-Fattah [8]. His results showed that, when the centrifugal force increases, the radial normal stresses and the shear stresses are increasing. The standard $k-\varepsilon$ model was used to study the turbulent slot jet impinging on a semicylindrical convex surface by Hwang and Yang [9]. The comparison of Yang- Shih and standard $\mathrm{k}-\varepsilon$ turbulence model was studied by El-Gabry and Kaminski [10]. They showed that the standard k- $\varepsilon$ turbulence model is betier at a high Reynolds number and it is not possible to conclude that one turbulence model is more accurate than another. Direct numerical simulation of turbulent heat transfer in plane impinging jet was studied by Hattori and Nagano [11]. Their results indicated that Nusselt number increases with a decrease of the distance between impingement wall and the nozzle exit. Merci and Dick [12] used the cubic $k-\varepsilon$ model to study the heat transfer for axisymmetric turbulent jets impinging on a flat plate. Their results showed that Nusselt number decreases from the peak value at stagnation point until reaching a minimum value and increases to a maximum value and then decreases again with increasing the downstream distance. Also the numerical calculation of Nusselt number using $\vec{v}^{2}$-f turbulence model is less than the experimental data after the stagnation region. In addition, the predications from two models ( $k-\varepsilon$ and $\hat{v}^{2}$-f models) approached with an increase the downstream distance. The heat transfer from a row of turbulent jets impinging on a stationary surface was investigated numerically by Salamah and Kaminski [13]. Sveningsson and Davidson [14] were used $\bar{v}^{2}$ $f$ turbulence models in vane passage flow. Manceau et al. [15] studied the turbulent heat transfer predictions using the $\bar{v}^{2}$-f model on unstructured meshes Coussirat et al. [16] studied the computational fluid dynamics modeling of impinging gas - jet systems. They showed the effect of the different values of the inlet turbulence level (2.5\%$18 \%$ ) on Nusselt number. Their results indicated that the Nusuelt number increases by increasing of the turbulence intensity. Also, at the low value of the intensity of inlet turbulence $(2.5 \%)$, the values of Nusselt number are under predict the values from experimental data. Baydar [17] measured the pressure and axial velocity for the flow in two dimensions in $\mathrm{r}-\mathrm{Z}$ axes of single and double impinging jet. Beitelmal et al [18] investigated the effect of inclination of an impinging two dimensional air jet on the heat transfer from a uniformly heated flat plate. Their measured data was in the range of $4000 \leq \operatorname{Re} \leq 12000,4 \leq \mathrm{h} / \mathrm{b} \leq 12$ and $40 \leq \theta \leq 90$ .They found that, for low values of inclination angle, the local Nusselt number on the uphill side from the maximum heat transfer point was insensitive to jet exit -to- plate spacing. Akansu et al [19] studied experimentally, the effect of the inclination of an impinging two dimensional slot jet on the heat transfer from preheated flat plate. Their results showed the effect of Reynolds number, the distance between the nozzle and the plate on the Nusselt number and the pressure coefficient. Their measured data was in the range of $2500 \leq \operatorname{Re} \leq 75000,2 \leq \mathrm{H} / \mathrm{b}$ $\leq 12$ and $0 \leq \theta \leq 45$. Tzeng et al. [20] investigated numerically the heat transfer under confined impinging turbulent slot jets for eight turbulence model including one standard and seven lowReynolds number $k-\varepsilon$ models. Validation results indicated the prediction by each turbulence model was found to depend on grid distribution (i-e they used the dimensionless distance from the wall $\left(y^{+}\right)$ varying from 2,3 and 6 ) and numerical scheme used spatial discretization. The exist of spent fluid was set between impinging jets to reduce the cross-flow effect in degradation of the heat transfer of downstream impinging jets.

The aim of this numerically study is to investigate the effect of the distance between nozzles, Reynolds number and the flow time on the characteristics of unsteady, two dimensional and turbulent opposed jets. These characteristics are the velocity vectors, axial component, radial component, axial normal stress, turbulence kinetic energy and pressure coefficient. 


$$
\begin{aligned}
& \frac{\partial \bar{v}^{2}}{\partial t}+u \frac{\partial \bar{v}^{2}}{\partial r}+v \frac{\partial \bar{v}^{2}}{\partial y}=\frac{1}{r} \frac{\partial}{\partial r}\left[r\left(\frac{1}{\operatorname{Re}}+\frac{1}{\sigma_{\varepsilon} \operatorname{Re}_{i}}\right) \frac{\partial \bar{v}^{2}}{\partial r}\right] \\
& +\frac{\partial}{\partial y}\left[\left(\frac{1}{\operatorname{Re}}+\frac{1}{\sigma_{s}}\right) \frac{\partial \vec{v}^{2}}{\partial y}\right]+\rho k f-6 \rho \bar{v}^{2} \frac{\varepsilon}{k} \\
& f=L^{2} \frac{\partial^{2} f}{\partial r^{2}}+\frac{L^{2}}{r} \frac{\partial f}{\partial r}+L^{2} \frac{\partial^{2} f}{\partial y^{2}}+\left(c_{1}-1\right) \\
& \frac{\left(\frac{2}{3}-\frac{\bar{v}^{2}}{k}\right)}{\tau}+c_{2} \frac{G}{k}
\end{aligned}
$$

The expression for eddy viscosity is $\mu_{t}=c_{\mu} \rho \bar{v}^{2} \tau$ where $\tau$ is a time scale and $L$ is a length scale, which are calculated from the following relations:

$$
\begin{aligned}
& \tau^{*}=\max \left(\frac{k}{\varepsilon}, 6 \sqrt{\frac{v}{\varepsilon}}\right) ; \\
& \tau=\min \left(\tau^{*}, \frac{\chi \vec{v}^{2}}{\sqrt{6} c_{\mu} \sqrt{S^{2}}}\right) ; \\
& \mathrm{L}^{*}=\mathrm{C}_{\mathrm{L}} \max \left(\frac{k^{3 / 2}}{\varepsilon}, c_{\eta}\left(\frac{v^{3}}{\varepsilon}\right)^{1 / 4}\right)
\end{aligned}
$$

and

$\mathrm{L}=\min \left(L^{*}, \frac{1}{\sqrt{6}} \frac{\bar{v}^{2} k^{2 / 3}}{c_{\mu} \sqrt{S^{2}}}\right)$

The closure coefficient for the model as published in [14], are given as

$$
\begin{aligned}
& \chi=0.6, c_{1}=1.4, c_{2}=0.3, c_{\varepsilon 1}=1.4, c_{\varepsilon 2}=1.9, \\
& c_{\eta}=85, c_{\mu}=0.22 \quad c_{L}=0.25, \sigma_{k}=1.0, \sigma_{\varepsilon}=1.3 \\
& \quad \text { and } c_{\varepsilon 1}^{*}=1.4\left(1+0.045 \sqrt{\frac{k}{\bar{v}^{2}}}\right)
\end{aligned}
$$

The computational domain boundaries are shown in Fig. 1(b).

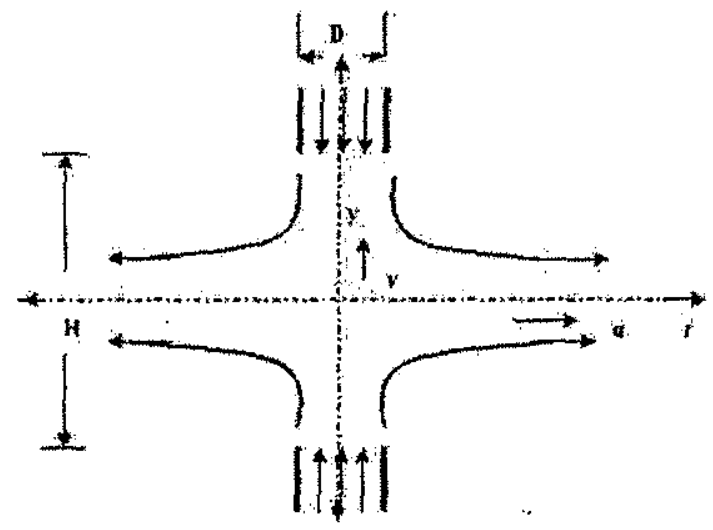

Fig, 1(a) Schematic diagram of opposed jet

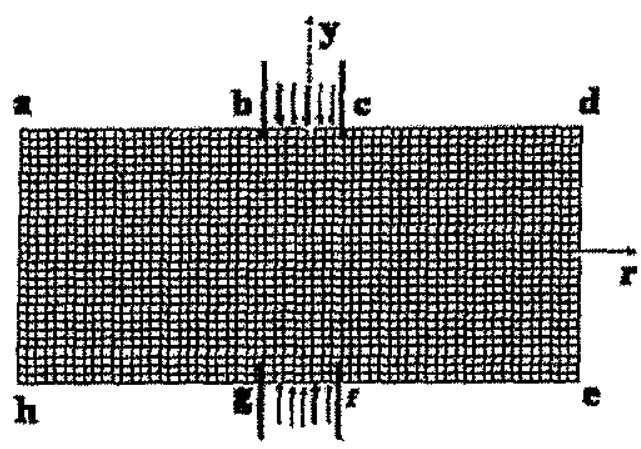

Fig. 1 (b) Computational domain

The boundary conditions for the above set of governing equations are as follows:

a) Inlet boundary $(b-c)$ and $(g-f)$

The uniform velocity profiles and turbulent kinetic energy dissipation rate are

$$
\begin{gathered}
\text { i.e., } v=V_{J}, T=T_{J_{1}} \quad k_{J}=0.003 V_{J}^{2}, \\
\varepsilon_{J}=\frac{c_{\mu} k_{J}^{1.5}}{0.06 b_{J}}, \quad \vec{v}_{j}^{2}=k_{j} \text { and } f_{j}=0.0
\end{gathered}
$$

b) at the axis

The boundary conditions correspond to asymmetry conditions at the axial and radial planes of symmetry so that for example, the axial velocity and all gradients of the other variables were zero at the stagnation plane,. In addition, the radial velocity and all gradients of the other variables were zero at the axial axis.

d) Exit boundary ( $a-b, c-d, d-e$ e-f, g-h and $h-a$ )

A zero gradient condition is employed for the outlet boundary. Although this boundary condition is strictly valid only when flow is fully developed, it is also permissible for sufficient downstream from the region of interest, i.e.,

$$
\frac{\partial \phi}{\partial r}=\frac{\partial \phi}{\partial y}=0 \text { and } \phi=u, v, \bar{v}^{2}, k \text { and } \varepsilon
$$

\section{SOLUTION PROCEDURE}

The mathematical models described above consist of a set of differential equations subject to appropriate boundary conditions. To provide the algebraic form of the governing equations, a fully staggered grid system was adopted for the velocity components and the scalar variables. These equations were discretized using a control volume finite difference method (CVFDM). The numerical solution in the present work is accomplished by using Semi- Implicit Method for pressure linked equation (SIMPLE) utilized by Patankar [21]. In the time step of duration dt, the velocity components $\mathrm{v}$ is calculated at the north and south faces of the main control volumes from the solution of the axial 


\section{A. Abdel-Fattah, "Numerical Study of Flow in Axisymmetric Turbulent Opposed Jets"}

momentum equation. Similarly, the velocity component $u$ at east and west face is calculated. When the pressure correction equation is solved, then the velocities and the pressure fields are corrected. To complete iteration, the turbulent kinetic energy and energy dissipation rate are solved successively. The discretization equation were solved by the line by- line procedure, which is a combination of GaussSeidel and tridiagonal matrix algorithm in the stream wise direction. The tridiagonal matrix algorithm (TDMA) was used to solve a set of discretization equation in the cross - equations in the cross- stream direction. Relaxation factors were employed to promote smooth convergence of the discretized equations. The relaxation factors were $0.75,0.75,0$. $5,0.5,0.1$ and 0.1 for $\mathrm{v}, \mathrm{u}, p^{\prime}, \mathrm{k}, \varepsilon$ and $\bar{v}^{\prime 2}$ respectively. The turbulent viscosity was under relaxed at a value of 0.1 . The converged criterion in this study was based on the successive changes in variables. All field variables were monitored, and the following condition was used to declare convergence:

$$
M A X\left|\frac{\phi_{i, j}^{n}-\phi_{i, j}^{n-1}}{\phi_{i, j}^{n}}\right| \leq 10^{-4}
$$

In addition, the ratio of the difference between the inlet mass flow rate and the outlet mass flow rate to the inlet mass flow rate was also examined. Convergence was declared if the relative mass imbalance was less than $10^{-3}$ and $\mathrm{Eq}$. (12) were satisfied simultaneousiy. After the convergence the flow at this time step, the entire variables are taking the initial condition for the new time step. To verify the algorithm, numerical tests were performed to ensure that the solution was grid algorithm.

The grid points are distributed uniformly over the computational domain.. A $128 \times 100$ grid points were placed in the computational domain in Fig.1(b). Results at a grid independent study are shown in Fig. 2.

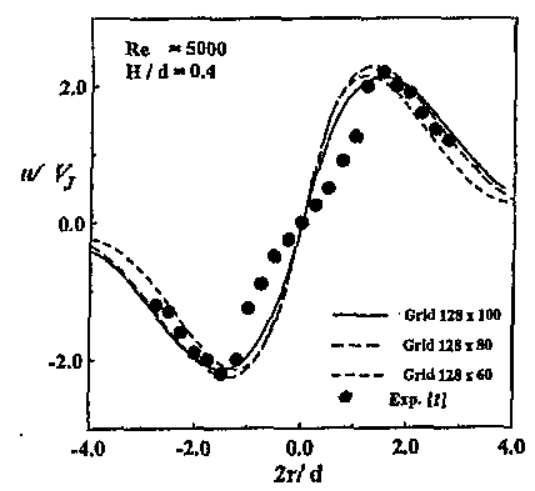

Fig. 2 Effect of grid refinement on the radial velocity along the separation plane using $v^{\prime 2}-f$ model

\section{RESULTS AND DISUSSION}

\subsection{The mean flow vector fields}

Figures $3 a-3 d$ illustrate a vector representation of the mean velocity fields for different values of the time at $\mathrm{Re}=5000$ and the distance between the nozzles $(\mathrm{H} / \mathrm{d}=0.4)$. In each case the flow is apparent, the flow issues from the two opposed nozzles at same axis. The flow spreads in the radial direction, combining at the separation plane and deflects in the radial direction. Also, from this figure, it can be seen that the deflection of the flow is low and secondary flow in the entrainment zone is high at low time. The secondary flow in the entrainment zone decays and the mean flow in radial direction increases by increasing the time. At high values of the time, the flow becomes constant. In this case, the flow becomes steady flow.

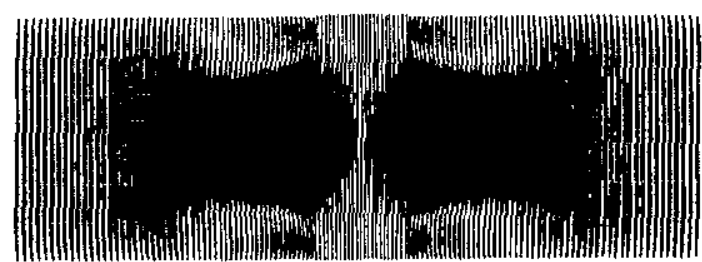

(a) $\bar{t}=0.01 \mathrm{sec}$

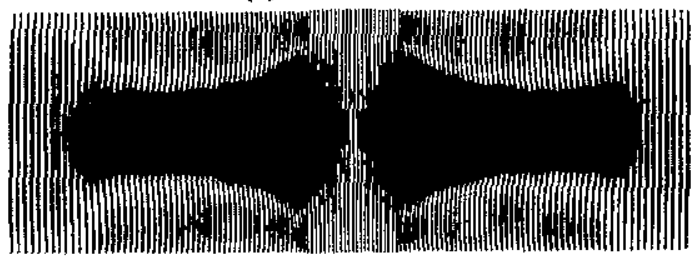

(b) $\vec{t}=0.03 \mathrm{sec}$

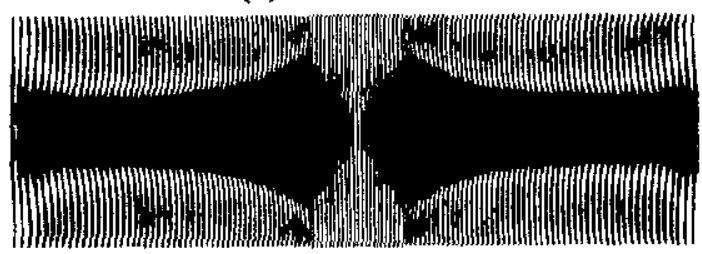

(c) $\bar{t}=0.05 \mathrm{sec}$

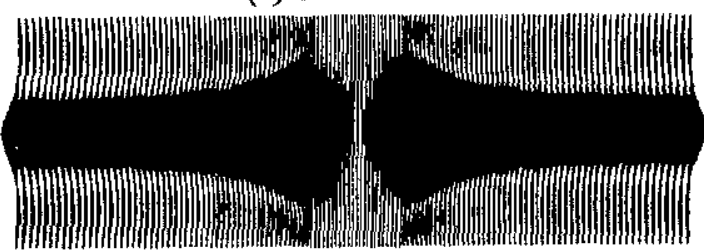

(d) $\bar{t}=0.1 \mathrm{sec}$

Fig. 3 Mean velocity vector field for different values of the flow time at the distance between the nozzles

$$
(\mathrm{H} / \mathrm{d}=0.4) \text { and } \mathrm{Re}=5000 \text { using } v^{\prime 2}-f \text { model }
$$

As the distance between the nozzles increases, the jet spreading increases in the radial direction. The flow combination of the two jets is delayed by increasing 
the distance between the nozzles, See figures $4 a-4 d$. Also the jet spreading after the defection increases with the radial distance increases. This spreading increases as the distance between the nozzles increases.

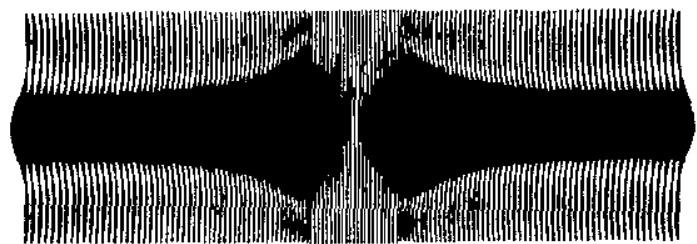

(a) $\mathrm{H} / \mathrm{d}=0.4$

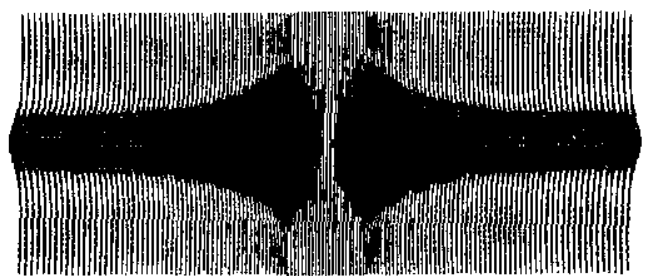

(b) $\mathrm{H} / \mathrm{d}=0.6$

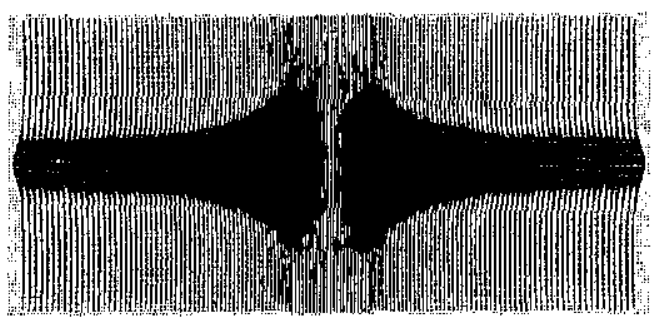

(c) $\mathrm{H} / \mathrm{d}=0.8$

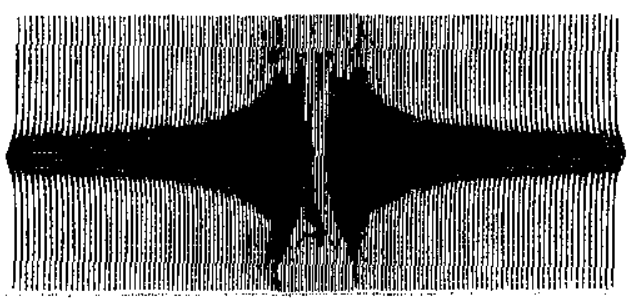

(d) $\mathrm{H} / \mathrm{d}=1.0$

Fig. 4 Mean velocity vector field for different values of the distance between the nozzles $(\mathrm{H} / \mathrm{d})$ at the time $\left(\bar{t}=0.2 \mathrm{sec}\right.$ ) and $\mathrm{Re}=5000$ using $v^{\prime 2}-f$ model

4.2. Effect of the distance between the nozzles on the radial velocity

Figure 5 shows the dimensionless radial velocity $\left(\mathrm{u} / \mathrm{V}_{\mathrm{J}}\right)$ as function of the radial distance at different values of the distance between the nozzles $(\mathrm{H} / \mathrm{d})$, constant $\mathrm{Re}=5000$, figure $5 \mathrm{a}$ at the axial distance $\mathrm{y} / \mathrm{d}$ $=0.06$ from the nozzle exit, figure $5 \mathrm{~b}$ and figure $5 \mathrm{c}$ on the radial axis. From this figure, it can be seen, the main case of asymmetry was the exit profile, which is increased with reduction in separation for all cases of the distance between the nozzles $(\mathrm{H} / \mathrm{d})$, see figures 5a. The velocity decreases by increasing the distance between the nozzles $(\mathrm{H} / \mathrm{d})$, This is caused by the jet spread increasing. The variation of this velocity along the radial axis is shown in figures $5 \mathrm{~b} \& 5 \mathrm{c}$. The general trend for each value of the distance between the nozzles was as an increase in the radial reaching to maximum value and it decays with the radial distance increases. This velocity increases accuses the combining of the two jets. The mean flow converts from the axial direction into the radial direction due to the flow combining. As the distance between the nozzles increases, the velocity decreases. This is because the intensity of flow decreases.

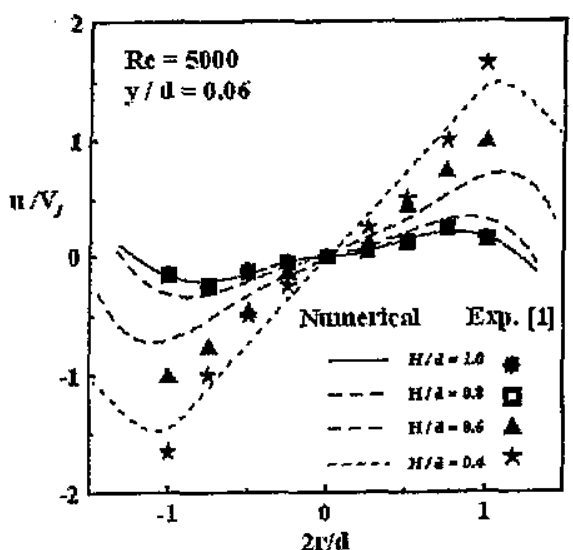

(a)

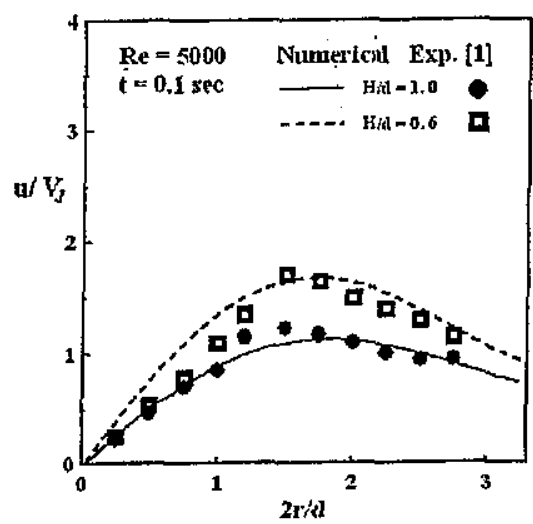

(b)

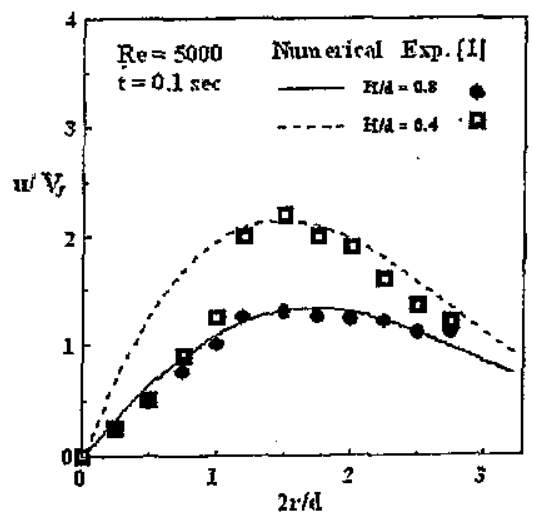

(c)

Fig. 5 Variation of the radial velocity with a nozzle separation $(\mathrm{H} / \mathrm{d}$ ) at $\mathrm{Re}=5000$ (a) at $\mathrm{y} / \mathrm{d}=0.06$ from the nozzle exit (b\& c) along the separation plane using $v^{\prime 2}-f$ model 


\subsection{Effect of the flow time on the radial velocity}

Figure 6 represents a graph of the dimensionless radial velocity $\left(u / V_{y}\right)$ as function of the radial distance at different values of the time $(\bar{t})$, a fixed constant $\mathrm{Re}=5000$, and the distance between the nozzles $(\mathrm{H} / \mathrm{d}=1)$. From this figure, it is noticed that, same trend for each value of the time but the increment of the value of the velocity decays by increasing the time and it is finished at higher value of the time and the flow becomes steady flow.

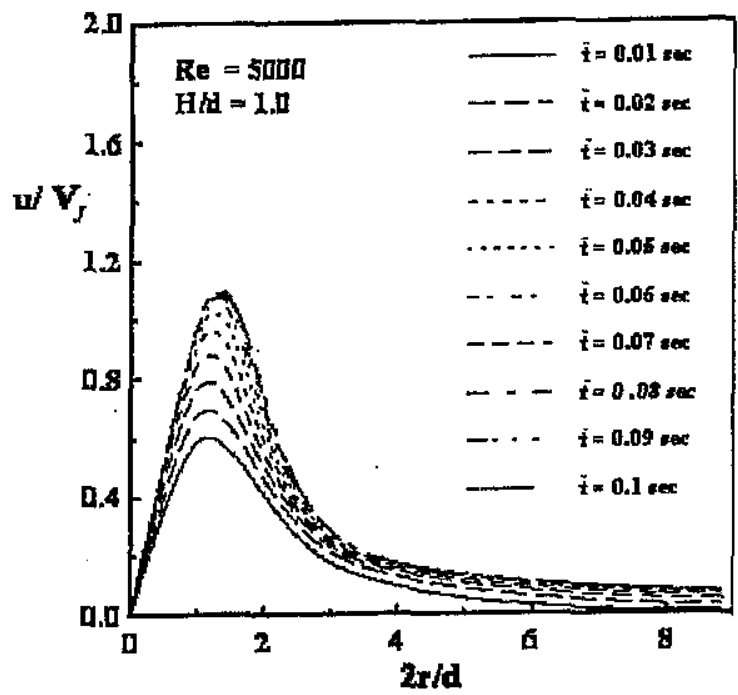

Fig. 6 Variation of the radial velocity with a time $(t)$ along the separation plane using $v^{\prime 2}-f$ mode

The effect of the turbulence model on the radial velocity is shown in figure 7 . The numerical results comparing with the experimental measurement [1] indicate that the $\bar{v}^{2}$-f model is better than RNG model and $\mathrm{k}-\varepsilon$ model.

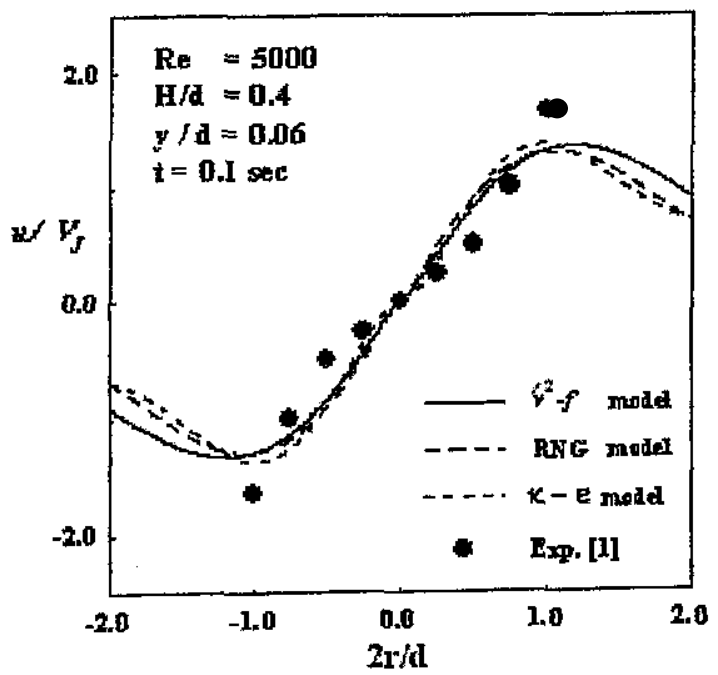

(a)

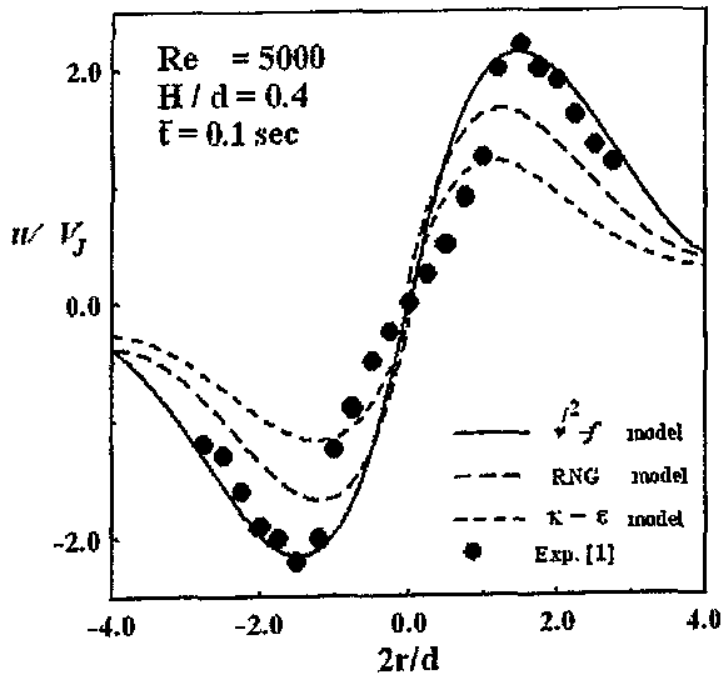

(b)

Fig. 7 Effect of turbulnce model on the radial velocity (a) at $y / d=0.06$ from the nozzle exit (b) along the separation plane

4.4. Effect of the distance between the nozzles on the axial velocity

The yariation of the dimensionless axial velocity $\left(\mathrm{v} / \mathrm{V}_{\mathrm{J}}\right)$ with the radial distance for several distance between the nozzles $(\mathrm{H} / \mathrm{d})$, at the distance from nozzle exit $\mathrm{y} / \mathrm{d}=0.06$ and constant $\mathrm{Re}=5000$ is given in figure 8. From this figure, it can be seen, the velocity increases from center of the jet and decreases in the radial direction reaching zero value in the both side of the jet. The value of this velocity decreases by increasing the distance between the nozzles. This is because the back effect for combination of the two jets becomes strong.

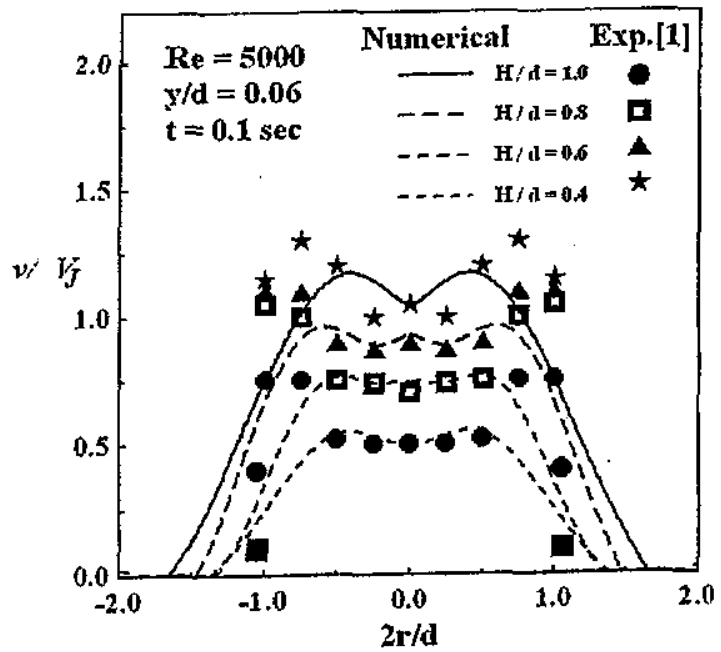

Fig. 8 Variation of the axial velocity with a nozzle separation $(\mathrm{H} / \mathrm{d})$ at $\operatorname{Re}=5000$ and $\mathrm{y} / \mathrm{d}=0.06$ from the nozzles exit using $v^{\prime 2}-f$ model 


\subsection{Effect of the flow time on the axial velocity}

Figure 9 represents a graph of the dimensionless axial velocity $\left(\mathrm{v} / \mathrm{V}_{\mathrm{J}}\right)$ as function of the axial distance at different values of the time $(\bar{t})$, a fixed constant $\mathrm{Re}=5000$, and the distance between the nozzles $(\mathrm{H} / \mathrm{d}=1.0)$. From this figure, it is noticed that, the velocity is maximum at the nozzle exit and decreases in the axial direction in the side of stagnation line, which occurs on the radial axis. This is due to combination of the two jets and inlet boundary condition occurred in the axial axis. Also it can be noticed that, the effect of the time on the axial velocity is low. This is because the change of the axial velocity in axial direction is faster accuses the axial direction is major.

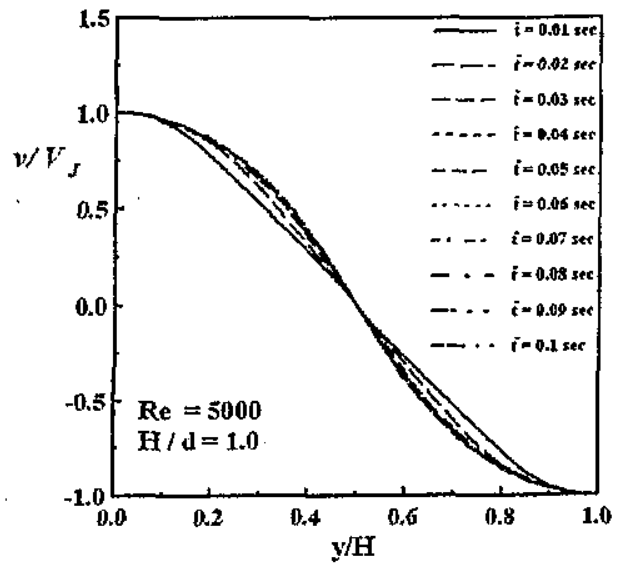

Fig. 9 Variation of the axial velocity with a time $(\mathrm{t})$ along the separation plane using $v^{\prime 2}-f$ model

The effect of the turbulence models on the axial velocity is shown in figure 10 . The comparison between the experimental measurement [1] and numerical results obtained from different turbulence models of $\mathrm{k}-\varepsilon$ is given in figure 10 . From this figure, it is seen that, the results from all models are approximately constant and are in good agreement with the experimental

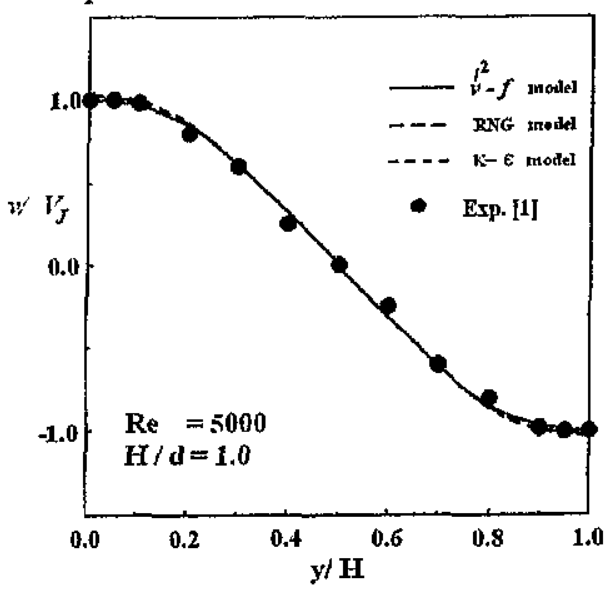

Fig. 10 Effect of turbulnce model on the axial velocity along the axis

\subsection{Effect of the distance between the nozzles on axial normal stress}

The variation of the axial normal stress along the axial direction $\left(v^{\prime} v^{\prime} / V_{J}\right)$ with the axial distance for various distance between the nozzles $(\mathrm{H} / \mathrm{d})$, at constant $\mathrm{Re}=5000$ is given in figure 11. From this figure, it is seen that the axial normal stress along the jet center increases in down stream direction in axial direction reaching the maximum value at stagnation point which occurs in the stagnation zone between the two jets and decreases in the direction of the other nozzle exit. This is due to combination of the two jets accuses the increase of the turbulence stresses. As the distance between the nozzles increases, the axial normal stress decreases. This is because the spreading jet increases as the distance between the nozzles increases, accordingly the decay of fluid flow increases due to jet spreading.

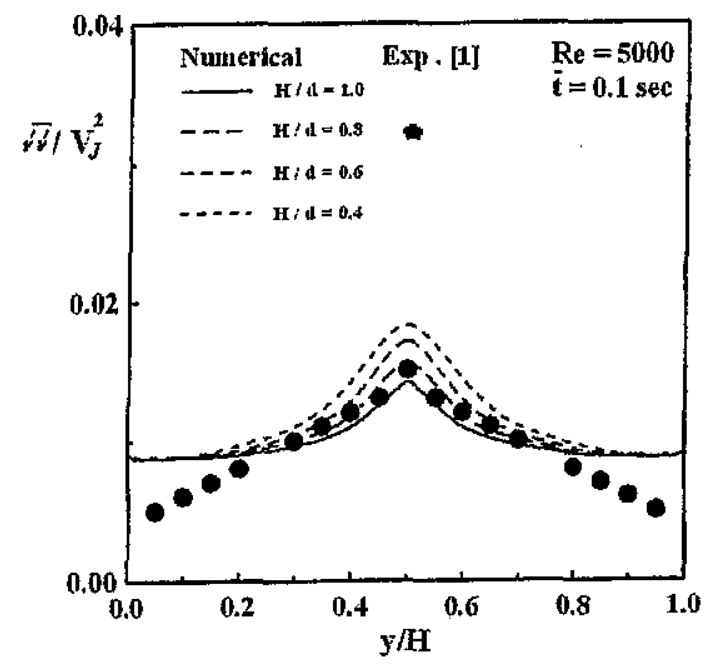

Fig. 11 Variation of the axial normal stress along the axis with a nozzle separation at $\mathrm{Re}=5000$

$$
\text { using } v^{\prime 2}-f \text { model }
$$

\subsection{Effect of the distance between the nozzles on turbulence kinetic energy}

Figures $12 \mathrm{a} \& 12 \mathrm{~b}$ declare the turbulence kinetic energy $\left(\mathrm{k} / V_{J}^{2}\right)$ for the various distance between the nozzles at constant $R e=5000$. From this figure, it is noticed that, the turbulence kinetic energy along the jet center increases in down stream in axial direction reaching the maximum value at stagnation line, which occurs in the separation plane between the two jets and decreases in the direction of the other nozzle exit. This is due to combination of the two jets accuses the increase in the turbulence intensity. By increasing distance between the nozzles, the turbulence kinetic energy decreases. This is because the spreading jet increases as distance between the nozzles increases, accordingly the decay of fluid flow increases and consequently lower the disturbance of 
the flow, see figure 12a. Same conclusion for the turbulence kinetic energy along the radial axis is given by figure $12 b$.

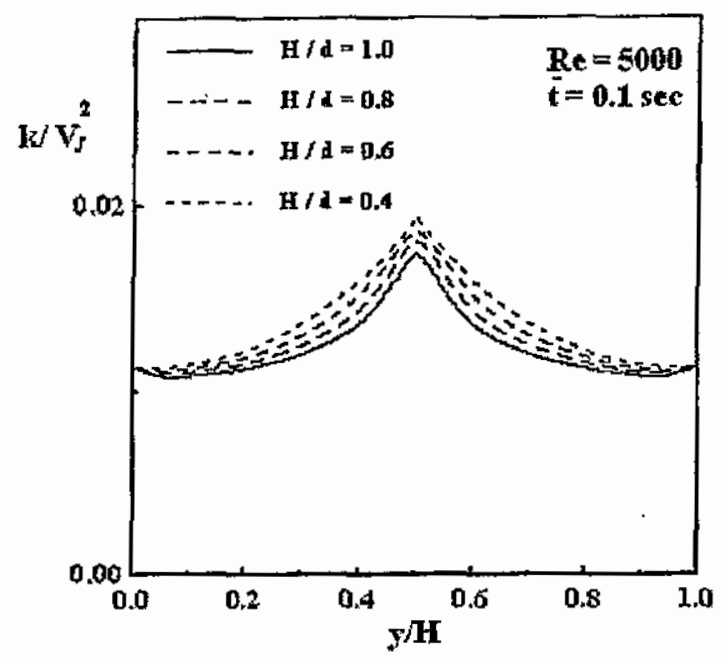

(a)

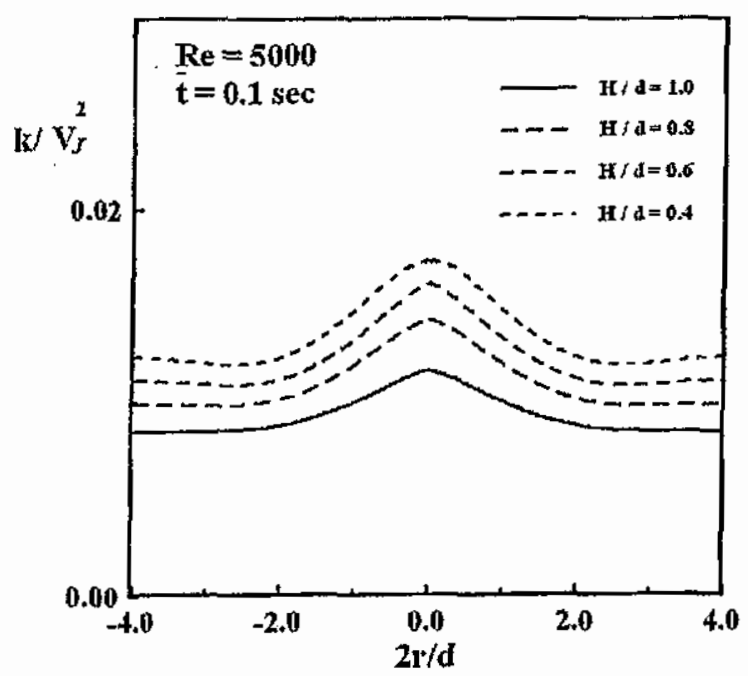

(b)

Fig.12 Variation of the turbulnt kinetic energy with a nozzle separation at $\operatorname{Re}=5000$ (a) along the axial axis (b) along the radial axis using $v^{\prime 2}-f$ model

The effect of changing the turbulence model on the turbulence kinetic energy for two values of Reynolds number is shown in figures $13 \mathrm{a} \& 13 \mathrm{~b}$. It is noticed that, the turbulence kinetic energy from the $k-\varepsilon$ model in stagnation zone is higher than the other models this because the production term in this model is higher .Also, the numerical results comparing with the experimental measurement [1] indicate that the $\bar{v}^{2}$-f model is better than RNG model and k- $\varepsilon$ model.

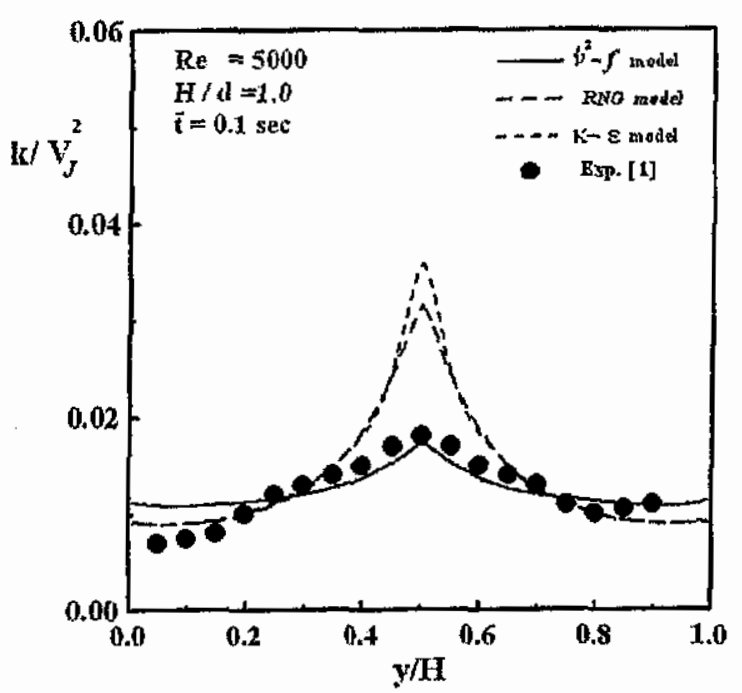

(a)

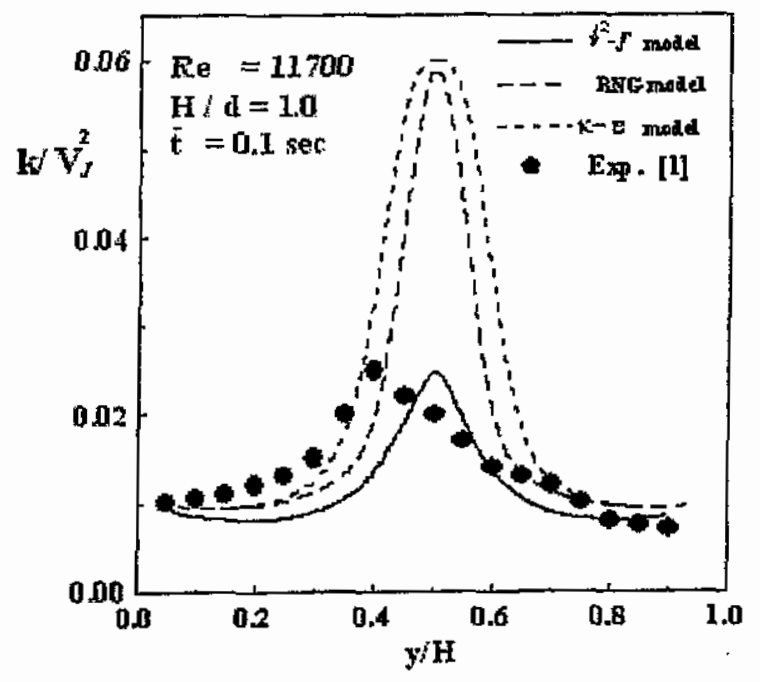

(b)

Fig. 13 Effect of turbulnce model on the turblunt kinetic energy along tha axial axis (a) at $\operatorname{Re}=5000$ \& (b) at $\operatorname{Re}=11700$

4.8. Effect of the distance between the nozzles on the pressure

Figure 14 indicates the dependence of the pressure coefficient $\left(\bar{p} / 0.5 \rho V_{J}^{2}\right)$ on the radial axis for different values of the distance between the nozzles $(\mathrm{H} / \mathrm{d})$, at constant $\operatorname{Re}=5000$. From this figure, it can be noticed that, the pressure coefficient is maximum and it is constant at the stagnation point for all cases of the distance between the nozzles. This point occurs at centerline of the jet and decreases as the radial distance increases. This is due to combination of the two jets accuses the stagnation the flow. The decay of the pressure coefficient from the stagnation point increases as the distance between the nozzles decreases. This is because the combining of the jets 
occurs early as the distance between the nozzles decreases.

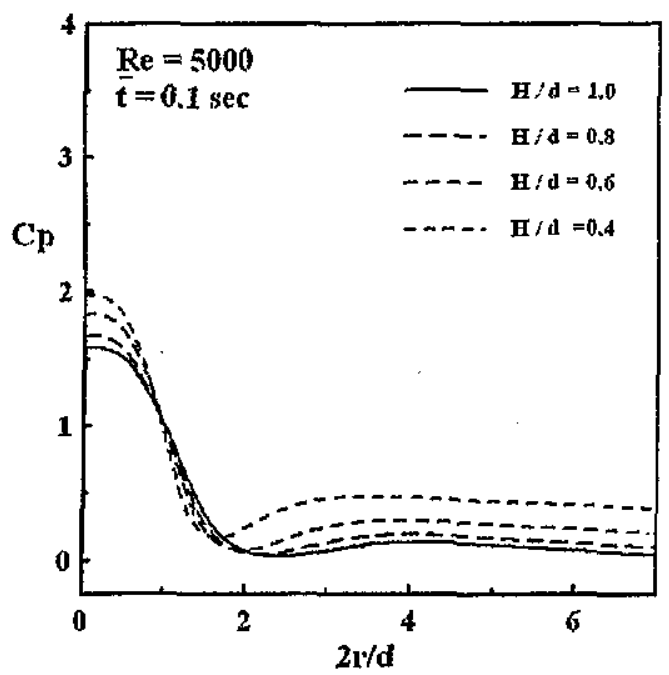

Fig. 14 Variation of the static pressure along radial axis with a nozzle separation at $=\operatorname{Re} 5000$

$$
\text { using } v^{\prime 2}-f \text { model }
$$

\subsection{Effect of the flow time on the pressure}

Figure 15 represents a graph of the pressure coefficient $\left(\bar{p} / 0.5 \rho V_{J}^{2}\right)$ as function of the radial distance at different values of the time $(\bar{t})$, a fixed constant $\mathrm{Re}=5000$, and the distance between the nozzles $(H / d=1.0)$. From this figure, it is seen that, the pressure coefficient increases in the combining zone, which it is around $1.5 \mathrm{~d}$ and reaching the maximum and becomes constant as the time increases. After this zone the pressure coefficient increases and decreases again until becomes approximately constant by increasing the time. This is because the flow becomes steady.

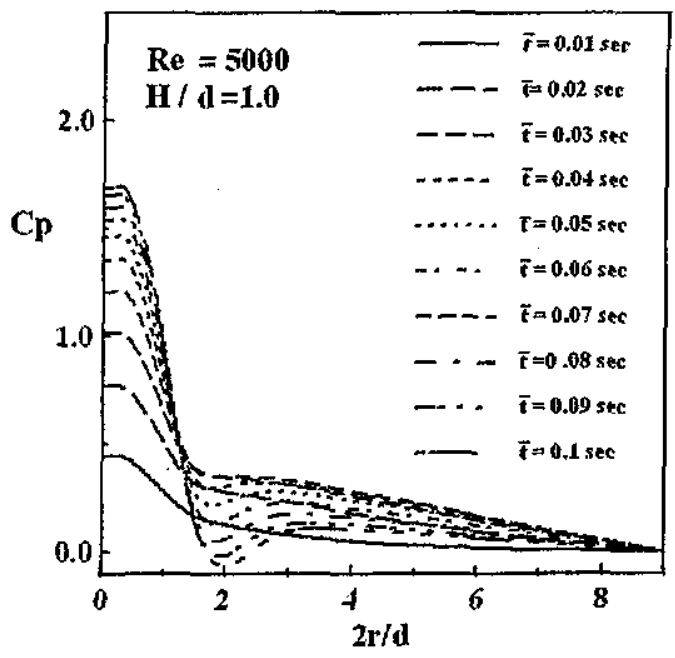

Fig. 15 Variation of the static the pressure along the radial axis with the time $(\vec{t})$ using $v^{\prime 2}-f$ model

\section{CONCLUSION}

The behavior of unsteady incompressible turbulent opposed jets flow field was carried out numerically.

The effect of the distance between the nozzles, Reynolds number, the flow time and the comparison between the turbulence models on the flow characteristics are studied. These characteristics are the velocity vectors, axial component, radial component, axial normal stress, turbulence kinetic energy and pressure coefficient

The major conclusions of this research are summarized as fallows:

- The velocity vectors fields depends on the flow time. As the time increases, the deflection of the flow from the axial direction into the radial direction increases also the intensity of the secondary flow, which occurs on the sides of the main flaw decreases. The flow becomes steady at higher values of the time.

- The jet spreading increases as the distance between the nozzles increases.

- The axial velocity decreases in the down stream direction and it reaches a zero at the stagnation point which occurs on the separation plane. As the time increases, the change of the values of this velocity is less and it is zero at higher time.

- The radial velocity increases in the down stream direction and it reaches a maximum value and then decreases. By increasing the distance between the nozzles, the value of this velocity decreases. As the time increases, the increment of the velocity value increases and this increment decreases to reach a minimum at higher value of the time.

- As the distance between the exit of the nozzles increases, the axial normal stress along the axial direction decreases. It becomes a maximum value at the stagnation point on the separation plane.

- The turbulence kinetic energy decreases by increasing the distance between the exits of the nozzles along the axial and radial directions

- The decay of the pressure coefficient from the stagnation point decreases by increasing the distance between the exits of the nozzles.

- The numerical results comparing with the experimental measurement [1] indicate that the $\bar{v}^{2}$.f model is better than RNG model and k- $\varepsilon$ model.

\section{NOMENCLATURE}

$c_{l}, \quad c_{2}, c_{d}, \quad$ empirical constants of $\mathrm{k}-\varepsilon$ model

$\mathrm{Cp} \quad$ pressure coefficient $\left(\bar{p} / 0.5 \rho V_{J}^{2}\right)$ 


\section{A. Abdel-Fattah, "Numerical Study of Flow in Axisymmetric Turbulent Opposed Jets"}

d nozzle inlet diameter mm dimensionless of variable related to energy redistribution in the equation of the scalar velocity scale $\bar{v}^{2}$

variable related to energy redistribution in the equation of the scalar velocity scale $\bar{v}^{2}$ $\sec ^{-1}$

centerline height between the exit of two nozzles mm

dimensionless of turbulent kinetic energy $\left(\vec{k} / V_{J}^{2}\right)$

$\bar{k} \quad$ turbulent kinetic energy $\quad \mathrm{J} / \mathrm{kg}$

$l$ dimensionless of length scale used in the $\bar{v}^{2}-\mathrm{f} \operatorname{model}(\bar{L} / \mathrm{d})$

$\bar{L} \quad$ length scale used in the $\bar{\nu}^{2}$-f model mm

$\mathrm{L}^{*}$ characteristic length used in the $\bar{v}^{2}$-f model

$\mathrm{P} \quad$ dimensionless pressure

$\bar{p}$

$\operatorname{Re}$

$R e_{t}$

$S_{i j}$

t

$\mathfrak{u}$

$\bar{u}$

static pressure $\mathrm{N} / \mathrm{m}^{2}$

Reynolds number $\left(R e=\rho V_{J} d / \mu\right)$

turbulent Reynolds number $\left(R e_{t}=\rho V_{J} d / \mu_{t}\right)$

deformation rate tensor

dimensionless flow time

dimensionless of radial component of local mean

radial component of local mean velocity $\mathrm{m} / \mathrm{sec}, \quad$ velocity $\left(\mathrm{u}=\bar{u} / V_{J}\right)$

dimensionless of axial component of local mean velocity $\left(v=\bar{v} / V_{J}\right)$

$\bar{v} \quad$ axial component of local mean velocity $\mathrm{m} / \mathrm{sec}$

average velocity at the jet injection location $\mathrm{m} / \mathrm{sec}$

dimensionless of turbulent velocity

$\vec{v}^{2} \quad$ scale $\left(v^{\prime 2}=\vec{v}^{\prime 2} / V_{J}^{2}\right)$

$\mathrm{I}, y$

cylindrical coordinates

dimensionless of dissipation rate of $k$

$\left(\varepsilon=\bar{\varepsilon} d / V_{j}^{3}\right)$

$\bar{\varepsilon}$

dissipation rate of $\mathrm{k}$

$\mathrm{J} / \mathrm{kg}$

$v$

kinematics viscosity

density

$\mathrm{kg} / \mathrm{m} 3$

model constant

model constant

dimensionless of turbulent time scale $\left(\bar{\tau} V_{J} / d\right)$

characteristic turbulent time scale used in the $\bar{v}^{2}-\mathrm{f}$ model

\section{Subscripts}

j jet

$\mathrm{t}$ indices for turbulent

\section{REFRENCES}

[1] Lindstedt, R .p., Luff, D.S. and Whitelaw, J.H., "Velocity and strain rate characteristics of opposed isothermal flows", Turbulence and Combustion 2002; 74:169-194

[2] Yang, H Lm, Kang Y. Huh and Kwang-yong $\mathrm{Kim}$, "Analysis of impinging and countercurrent stagnating flows by Reynolds stress model". Journal of fluid engineering 2002; 124: 706-718

[3] Luff, D, Korusoy, E. Lindstedt, R.P.and Whitelaw, J.H., "Counter Flow flames of air and methane, propane and ethylene, with and without periodic facing", Exp. Fluids 2003; 35(6): 618626.

[4] Korvsoy, E and Whitelaw, J.H, "inviscid laminar and turbulent flows" Int. J. Numerical Methods in Fluids 2004: 46:1069-098.

[5] Park, T.S. and Sung, H.J. "Development of a near wall turbulence model and application to jet impingement heat transfer", Int. J. Heat Fluid Flow 2001; 22:10-18

[6] Chattopadhyay, H.," Numerical investigation of heat transfer from impinging annular jet", Int. J. of Heat and Mass Transfer, 2004; 47: 3197- 3201

[7] Salmanzadeh, M. Mansouri, S., H., and Farhadi, M., "Numerical study of flow and thermal fields in an axisymmertic turbulent jet impinging on a flat plate" International Mechanical Engineering Conference, Kuwait, December,4-8, 2004: 200208

[8] Abel-Fattah,A," Numerical simulation of turbulent impinging jet on a rotating disk", Int. J. Numerical Methods In Fluids 2007; 53(11):16731688

[9] Hwang, C. H.and Yang,Y..T, "Numerical simulations on the hydrodynamics of a turbulent slot jet impinging on a semi cylindrical convex surface" Numerical Heat Transfer Part A. 2004; 46: 995-1008.

[10] El-Gabry, L. A. and Kaminski, D.A.., "Numerical investigation of Jet impingement with crass flow-comparison of Yang-Shih and standard k $\varepsilon$ turbulence models", Numerical Heat Transfer Part A. 2005; 47: 441-469

[11] Hattori $H$ and Nagano $Y .$, "Direct numerical simulation of turbulent heat transfer in plane impinging jet", Int. J. of Heat fluid flow 2004; 25 : 749-758. 
[12] Merci, B. and Dick E., "Heat transfer predictions with a cubic $k-\varepsilon$ model for axisymmetric turbulent jets impinging on to a flat plate", Int. J. of Heat Mass Transfer 2003; 46: 469-480

[13] Salamah S. A., and Kaminski, D.A., "Modeling of turbulent heat transfer from an Array of submerged jets impinging on a solid surface ",Numerical Heat Transfer Par A-2005; 48:. 315337.

[14] Sveningsson, A., and Davidson, L.," Assessment of realizability constraints in $\bar{v}^{2}-f$ turbulence models", International Journal of Heat fluid flow 2004; 127: 785-794

[15] Manceau,R. Parneiex,S. and Laurence, D.," Turbulent heat transfer predictions using $\bar{v}^{2}$-f on unstructured mashes", Int. J. of Heat and Fluid Flow 2000; 21: 320-328.

[16] Coussirat, M., Van Beeck, J., Mestres, M.Egusguiza, Buchlin, J. M. and Escaler, E., "Computational fluid dynamics modeling of impinging gas-jet systems I assessment of eddy viscosity models", Journal of Fluids Engineering 2005; 127: 691703

[17] Baydar,E., "Confined impinging air jet at low Reynolds numbers" Experimental Thermal Fiuid Science, 1999; 19: 27-33

[18] Beitelmal A. H., Saad, M.A., and Potel,C ,D. "The effect of inclination on the heat transfer between a flat plate and an impinging two dimensional air jet", Int. J. of Heat-Mass Transfer 2000; 21: 156-163.

[19] Akansu, Y.E., Sarioglu, M.and Yavuz, T., "Heat transfer characteristics of a slot jet impinging on a flat plate at angle of incidence", Third Int.Conference on Energy Research and Development (ICERD-3) Novamber 21 23, 2005 (Kuwait).

[20] Tzeng, P. Y.Soong, C.Y. and Hsieh, C.D. "Numerical investigation of heat transfer under confined impinging turbulent slot jets", Numerical Heat Transfer Part A. 1999; 35: 441-469

[21] Patankar, S.V., "Numerical heat transfer and fluid flow" McGraw-Hill, New York, 1980. 